\title{
Solving the Near-Far Problem in Dynamic Frequency Hopping-Optical Code Division Multiple Access using Power Control
}

\author{
Wamidh J. M. ALgalbi \\ Electrical network dep. \\ Technical Institute Shatra \\ Thi-Qar, Iraq \\ Wam_jaleel@yahoo.com
}

\begin{abstract}
In this study, a distributed power control algorithm is proposed for Dynamic Frequency Hopping Optical-CDMA (DFH-OCDMA) system. In general, the DFH-OCDMA can support higher number of simultaneous users compared to other OCDMA techniques. However, the performance of such system degrades significantly as the received power does lower than its minimum threshold. This may obviously occur in a DFH-OCDMA network with near-far problem which consist of different fiber lengths among the users, that resulting to unequal power attenuation. The power misdistribution among simultaneous active users at the star coupler would degrade the Bit Error Rate (BER) performance for users whose transmitting signals with longer fiber lengths. In order to solve these problems, we propose an adaptive distributed power control technique for DFH-OCDMA to satisfy the target Signal to Noise Ratio (SNR) for all users. Taking into account the noise effects of Multiple Access Interference (MAI), Phase Induced Intensity Noise (PIIN) and shot noise, the system can support $100 \%$ of users with power control as compared to $33 \%$ without power control when the initial transmitted power was $-1 \mathrm{dBm}$ with 30 simultaneous users.
\end{abstract}

Key words: Optical-CDMA, Dynamic Frequency Hopping-OCDMA, power control

\section{INTRODUCTION}

Since mid 1980's, optical communications have became very important in the world wide. Up to this date, many techniques have been researched in many fields of optical communication due to their attractive features such as vast bandwidth, high-speed signal processing and reliable security [1-4]. The techniques include optical code multiple access (OCDMA), introduced due to the fast growing demand of transmission bandwidth and system capacity. OCDMA provides users with asynchronous access of the medium by assigning each particular user with a specific code signature. Therefore huge number of simultaneous users can be shared optically with high reliability. Spectral Amplitude Coding (SAC-OCDMA) [10], Fast Frequency Hopping (FFH-OCDMA) [6], Modified Frequency Hopping (MFH) [2], and Dynamic Frequency Hopping (DFH-OCDMA) [6] are examples of OCDMA techniques that offer this high capacity. However, due to the multiple access transmission, these techniques suffer with the users' interference called as multiple access interference (MAI). The impact becomes worst in the system with different fiber lengths. The power misdistribution among simultaneous active users at the star coupler would degrade the Bit Error Rate (BER) performance for some users which transmitting signals through longer fiber lengths. Therefore there is a need to equalize the power at can be achieved by using power control

Power control is one of the key technologies that increase system capacity and quality effectively. Two approaches can be considered, namely, centralized power control (CPC) and distributed power control (DPC [8]. Both approaches are used to optimize the launched power and maximize network capacity based on the number of users satisfying a targeted signal to interference (SIR) ratio.

Power control is proposed previously for singlerate direct detection DS-OCDMA systems by using double optical hard-limiter correlation receivers [13]. This technique needs two threshold settings for the first and second hard limiters. These thresholds are generally 
dependent on the received optical power and the number of simultaneous users. In [7], power control is also proposed for multimedia networks with time hopping-OCDMA, based on diversity receiving with hard limiting.

It is noted that the power control technique has only be discovered for prime code [5] and FFH code [9] , [14],[15] even there would be more potential code that can be considered. This paper therefore proposes and investigates on how much the improvement can be obtained in DFH-OCDMA using power control especially when such system suffers the effects of nearfar problem.

\section{THE IMPORTANCE OF POWER CONTROL FOR DFH-OCDMA}

DFH-OCDMA is chosen as the scope of this study because of the following considerations. In general, DFH-OCDMA has been proposed as a solution to provide easy reconfiguration of the transmitter without the need for sophisticated encoder [6]. The encoder varies the central frequency of optical pulse signal according to the functional code set to the controller. It can be realized using controlled Tunable Optical Filter (TOF) in each encoder and decoder sides with sinusoidal function given in Eq. (1) [6].

$$
\mathrm{F}(\mathrm{t})_{\mathrm{i}}=\frac{\Delta \mathrm{v}}{2} \sin (2 \pi \mathrm{ft}-\mathrm{i} \theta) \quad \mathrm{i} \in(1,2 . ., \mathrm{K})
$$

where $\mathrm{K}=$ The number of simultaneous users, $\Delta \mathrm{v}=$ The bandwidth of the optical frequency, $f=$ The optical frequency, $\theta=$ The phase shift between two successive codes.

Another advantage of DFH-OCDMA is that, it can accommodate simultaneous users as maximum as 169 ${ }^{[6]}$, in average, twice as much as compared to other OCDMA techniques. Nevertheless, the BER deteriorated rapidly with the small reduction of the threshold received power compared to other techniques. The comparison of BER degradation between DFHOCDMA and other techniques is tabulated in Table 1. It is shown that, a little change of threshold received power (from $-10 \mathrm{dBm}$ to $-12 \mathrm{dBm}$ ) yields great BER degradation from $10^{-9}$ to $10^{-6}$ while the other techniques did not show significant changes. This phenomenon may significantly occur in a DFH-OCDMA network with near-far problem as shown in Fig. 1. In order to solve these problems, distributed power control technique for DFH-OCDMA is proposed that would strongly satisfy the target Signal to Noise Ratio (SNR) for all users.

Table 1: Comparison of DFH-OCDMA and other OCDMA techniques for BER degradation due to receiver sensitivity

\begin{tabular}{lll}
\hline \multicolumn{1}{c}{ Code } & \multicolumn{1}{c}{ Change of Pr. } & \multicolumn{1}{c}{ BER change } \\
\hline FFH & $-40 \rightarrow 0$ & $10^{-3} \rightarrow 10^{-3.2}(\approx$ Fix $)$ \\
SAC-Hadmard & $-40 \rightarrow 0$ & $10^{-1} \rightarrow 0.5 \times 10^{-1}$ \\
SAC-MQC & $-40 \rightarrow 0$ & $10^{-1} \rightarrow 10^{-4}$ \\
SAC-MFH & $-40 \rightarrow 0$ & $10^{-1} \rightarrow 10^{-4}$ \\
DFH-OCDMA & $-40 \rightarrow 0$ & $10^{-1} \rightarrow 10^{-12}$ \\
\hline
\end{tabular}

\section{DISTRIBUTED POWER CONTROL METHOD}

To apply power control with DFH-OCDMA, we need to initially analyze the noisy received signal. The received signal at decoder side for $K$ users can be divided into two parts: the signal coming from $i^{\text {th }}$ user which include the data and noise and second part represented the MAI which come from other users $K-1$, where $K$ is the total active users. In DFH-OCDMA case, the received signal $(\mathrm{Sr})$ is given by:

$$
S_{r}=S_{\text {desired }}+M A I
$$

where $S_{\text {desired }}$ is the desired signal, while MAI is the interference signal found from the Eq. 3[6]:

$M A I_{i}=\frac{p_{r}}{T_{b} \Delta v_{j}} \sum_{j=1, j \neq i}^{k} b_{j} \sum_{i=1}^{N_{i}, j}\left(\left(B W\left(\tau S_{i}(i, j)-\tau E_{i}(i, j)\right)-\sum_{\tau S_{j}(i, j)}^{\tau E_{j}(i, j)}\left|F_{j}(t)-F_{i}(t)\right| d t\right)\right.$

where $\operatorname{Pr}$ is the received power, $\Delta v$ is the bandwidth of the optical frequency, $\mathrm{T}_{\mathrm{b}}$ bit time duration, $\mathrm{BW}$ is the Bandwidth of the TOF, S and $\mathrm{E}$ are the start and end interference time between $i$ and $j$ users respectively, $\mathrm{F}_{\mathrm{j}}$, $\mathrm{F}_{\mathrm{i}}$ are the functions in (Eq. 1) of users $i$ and $j$ respectively, $\mathrm{N}_{\mathrm{i}, \mathrm{j}}$ is the intersecting points between users $i$ and $\mathrm{j}$ which is depend on phase shift between the users. This code was extensively discussed in[6]. 


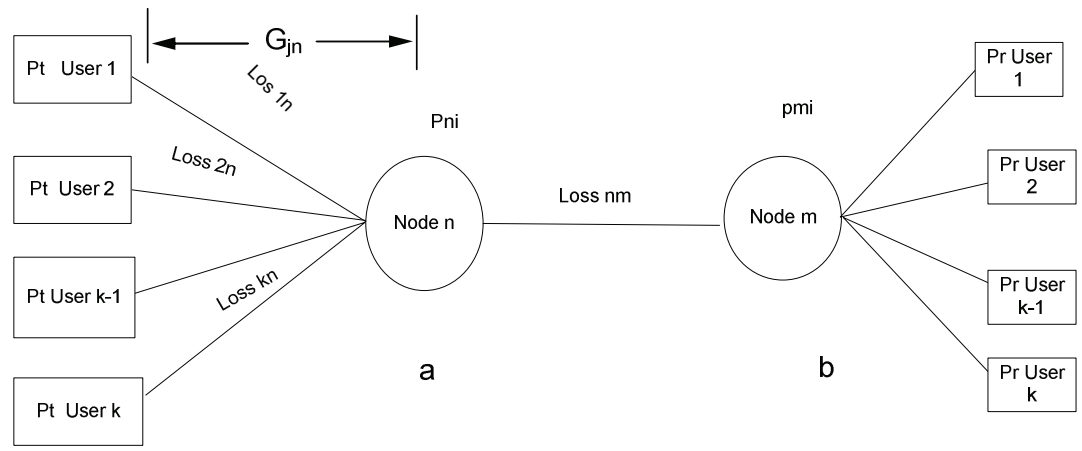

$G_{j n}$ : gain path for user $\mathrm{j}$ from node $\mathrm{n}$

Fig. 1: Star Network at Different Fiber Lengths for K users: (a) at Transmitter Side, (b) at Receiver Side.

MAI in Eq. 3 is the major problem in OCDMA as it limits the network at lower SNR. Instead of MAI, PIIN and shot noises also affect on the value of SNR.

Basically, the photocurrent variance due to PIIN is given by [10]:

$$
\sigma_{\text {PIIN }}^{2}=\mathrm{I}^{2} \tau_{\mathrm{c}} \mathrm{B}
$$

where $\mathrm{I}$ is the average photocurrent, $\tau_{\mathrm{c}}$ is the coherence time of the source, $\mathrm{B}$ is the noise equivalent electrical bandwidth.

In DFH-OCDMA, the PIIN variance for $K$ users is represented by [6]:

$$
\begin{aligned}
& P \text { IIN }=B \Re^{2}\left(\frac{\operatorname{Pri}}{\Delta v}+\frac{\operatorname{Prj}}{\Delta v}\right)^{2} \\
& \left(B W-\left|F_{i}(t)-F_{j}(t)\right|\right)+B \Re^{2}\left(\frac{P_{i}}{\Delta v}\right)^{2}\left|F_{i}(t)-F_{j}(t)\right|
\end{aligned}
$$

Meanwhile the basic variance of shot noise is represented as the following [11]:

$$
\underset{\sigma \text { shot }}{2} \sqrt{2 e B I}
$$

where $\mathrm{e}$ is the electronic charge, $\mathrm{B}=$ noise equivalent electrical bandwidth, I is the average photocurrent.

The value of I in DFH-OCDMA also depends on the received power over the bandwidth of TOF, $(\operatorname{Pr} / \Delta v)$ and therefore, the shot noise variance in DFH-OCDMA is considered as:

$$
\sigma_{s h i}^{2}=\sqrt{2 \Re e B \cdot B W \cdot \frac{p r_{i}}{\Delta v}}
$$

The Eq. 3, 5 and 7 in [6] assume that the received power is equally-value for all users. This is true only if all users transmit the signals at the same path length. Although the other parameters can be remained as constant since the bandwidth of TOF does not change with the power misdistribution, as far as the near-far problem is concerned, the Pr value in Eq. 3 can not be considered uniform. So the MAI for simultaneous users are not similar, or:

$\mathrm{MAI}_{1} \neq \mathrm{MAI}_{2} \neq \mathrm{MAIK}$

where $\mathrm{MAI}_{1}, \mathrm{MAI}_{2}$ and $\mathrm{MAI}_{\mathrm{K}}$ are the multi access interference for first user, second user and last user respectively and $K$ is number of simultaneous users. Noted that, the value of MAI for any user $i$ is found to be linearly proportion with $\operatorname{Pr}$ at another decoder $j$, thus the Eq. 3 can be simplified as the MAI of user $i$, given by:

$\mathrm{MAI}_{\mathrm{i}}=\mathrm{A} \cdot \operatorname{Pr} \mathrm{j}$

where $\mathrm{A}$ is a constant representing the design parameters of DFH-OCDMA in Eq. 3. The value of A depends on the bandwidth of the system, light speed, bandwidth of TOF and code size of the function of users defined in Eq. 1.

In the same way, the value of PIIN and shot noise variances in Eq. 5, 7 respectively will take new equations as follows:

$\operatorname{PIIN}_{i}={ }_{B} \sim{ }_{1}(\operatorname{Pri}+\operatorname{Prj})^{2}+{ }_{B} \sim{ }_{2}(P r i)$

$$
\stackrel{2}{\sigma} \operatorname{shi}=C 1 \cdot \operatorname{Pr} i
$$

where 


$$
\begin{aligned}
& \mathrm{B}^{\sim 1}=\frac{\mathrm{B}_{\Re}^{2}}{\Delta \mathrm{v}^{2}}(\mathrm{BW}-|\mathrm{Fi}(\mathrm{t})-\mathrm{Fj}(\mathrm{t})|), \\
& \mathrm{B}^{\sim 2}=\frac{\mathrm{B}_{\Re}{ }^{2}}{\Delta \mathrm{v}^{2}}(|\mathrm{Fi}(\mathrm{t})-\mathrm{Fj}(\mathrm{t})|), \text { and } C 1=2 \Re e B\left(\frac{B W}{\Delta v}\right)
\end{aligned}
$$

It is clear now; the MAI, PIIN and shot noises for all users are affected by received power of other users and varies linearly with $\mathrm{Pr}$. Since the $\mathrm{Pr}_{\mathrm{j}}$ in Eq. 9, 10 are different based on near-far problem, we need to take the sum average of $\operatorname{Pr}_{j}$ for all users. Rearranging Eq. 9, 10 and 11, the variance of each of MAI, PIIN and shot noise can be represented by:

$$
\begin{aligned}
& \sigma^{2} \mathrm{MAI}_{i}=\operatorname{Al}\left(\frac{1}{\mathrm{~K}-1} \sum_{\substack{j=1 \\
j \neq 1}}^{\mathrm{K}} \operatorname{Pr} \mathrm{j}\right) 2 \\
& \sigma^{2} \mathrm{PIIN}_{\mathrm{i}}=\mathrm{B} 1\left(\frac{1}{\mathrm{~K}-1} \sum_{\substack{j=1 \\
j \neq i}}^{\mathrm{K}} \operatorname{Pr} j\right) 2 \\
& \sigma^{2} \mathrm{sh}=\mathrm{C} 1 \cdot \operatorname{Pr} i
\end{aligned}
$$

where $\mathrm{A} 1, \mathrm{~B} 1$ and $\mathrm{C} 1$ are constants that lead to linear proportion relationship between MAI, PIIN and shot noises, and Pr respectively.

The SNR in OCDMA can now be represented by:

$$
\mathrm{SNR}_{i}=\frac{\mathrm{I}^{2}}{\sigma^{2} \mathrm{MAI}+\sigma^{2} \mathrm{PIIN}+\sigma^{2} \mathrm{sh}+\sigma^{2} \text { th }}
$$

where $\sigma^{2}$ MAI, $\sigma^{2}$ PIIN, $\sigma^{2}$ sh are the variances of MAI, PIIN, and shot noises as shown in Eq. 13, 14 and 15 respectively and $\sigma^{2}$ th is the thermal noise which is equal to :

$\sigma^{2}$ th $={ }^{4} \mathrm{~K}_{\mathrm{b}}{ }^{\mathrm{TB}} / \mathrm{R} \mathrm{L}$

where $\mathrm{K}_{\mathrm{b}}$ is the Boltzmann's constant, $\mathrm{T}$ is the Absolute receiver noise temperature, $T_{B}$ is the noise equivalent electrical bandwidth of receiver, $R_{L}$ is the receiver load resistor.

As a result, the SNR in DFH-OCDMA at various Pr with constant values of $\mathrm{A} 1, \mathrm{~B} \sim 1, \mathrm{~B} \sim 2$ and $\mathrm{C} 1$ is:

$$
\mathrm{SNR}_{i}=\frac{\mathrm{I}^{2}}{(\mathrm{~A} 1+\mathrm{B} 1)\left(\frac{1}{\mathrm{~K}-1} \sum_{\mathrm{j}=1}^{\mathrm{K}} \operatorname{Pr} \mathrm{j}\right)+\mathrm{C} 1 \operatorname{Pr}_{i}+\sigma^{2} \text { th }}
$$

The main purpose of applying power control is to reduce all these noises by adjusting appropriate transmitted power of certain users in such a way that all users may obtain the target SNR.

The SNR for $i^{\text {th }}$ user in Eq. 17 represents the first parameter for $\mathrm{PC}$ algorithm in Eq. 18 and this value will be compared with target $\mathrm{SNR}\left(\mathrm{SNR}_{\mathrm{tar}}\right)$ to estimate appropriate transmitted power for the next iteration (if any), shown as follows [12].

$$
\operatorname{Pr} i(n+1)=\frac{S N R t \arg }{S N R i(n)} \operatorname{Pr} i(n)
$$

where $n$ is the number of iteration of PC. The transmitted power is updated by scaling the current power level by the ratio between the target SNR and the current SNR. In the DFH-OCDMA case, the $\mathrm{SNR}_{\text {targ }}$ must be equal to $16 \mathrm{~dB}$ to achieve $\mathrm{BER}=10^{-9}$. If for example, the obtained SNR for $\mathrm{t}^{\text {th }}$ user is $15 \mathrm{~dB}$ at $n$th iteration, then the $\mathrm{Pt}_{\mathrm{i}}(\mathrm{n}+1)=1.06 \times \mathrm{Pt}_{\mathrm{i}}$ at $(n+1)$ th iteration, considering that the power vector does not exceed the initial transmitted power. A flowchart shown in Fig. 2 summarizes the power control algorithm.

\section{RESULTS AND DISCUSSION}

Numerical simulation has been conducted using Matlab ${ }^{\circledR}$ to model the proposed algorithm. The parameters used are: $\mathrm{BW}=1.65 \mathrm{~nm}, \mathrm{f}=1550 \mathrm{~nm}, \Delta \mathrm{v}=$ $30 \mathrm{~nm}$. The number of users considered is 100 , starting with fiber length of $0.2 \mathrm{~km}$ for the first user, up to 20 $\mathrm{km}$ for the last user. In this case, the difference of fiber length among adjacent user is $0.2 \mathrm{~km}$. The system is considered as fully supported if the system can support all simultaneous users. In Fig. 2, it was observed that without power control, the system was not fully supported (i.e only 77 out of 100 users managed to obtain BER of $\left.10^{-9}\right)$. After the application of power control in the first iteration, the system has improved to support 94 number of users. It is then gradually supported 100 percent of users after the second iteration. 


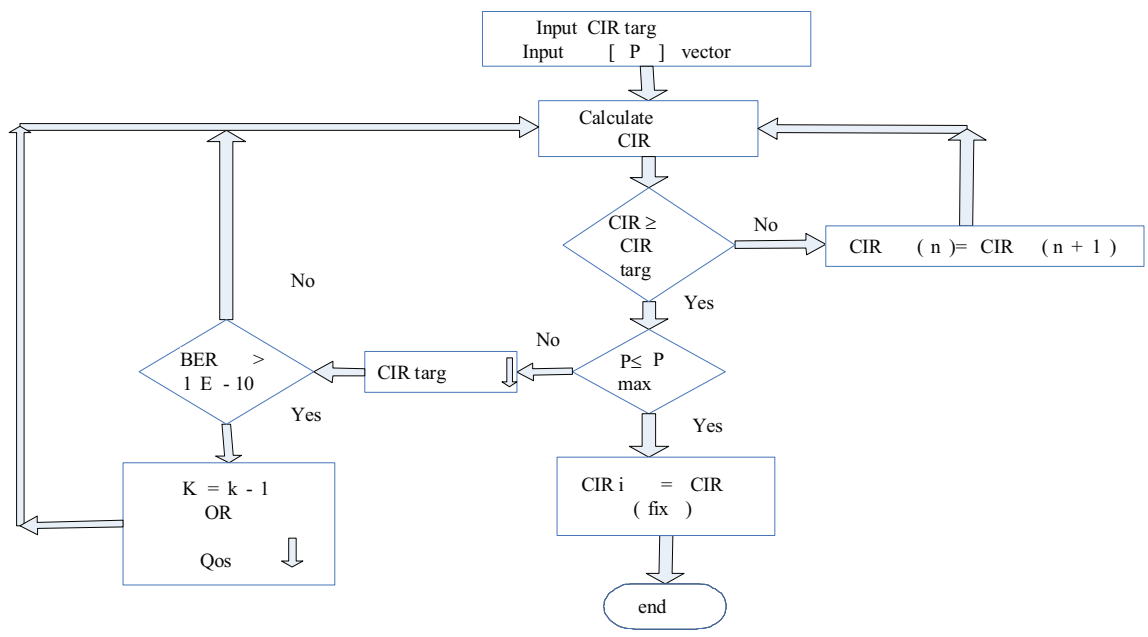

Fig. 2: Flowchart to Apply Power Control with DFH-CDMA

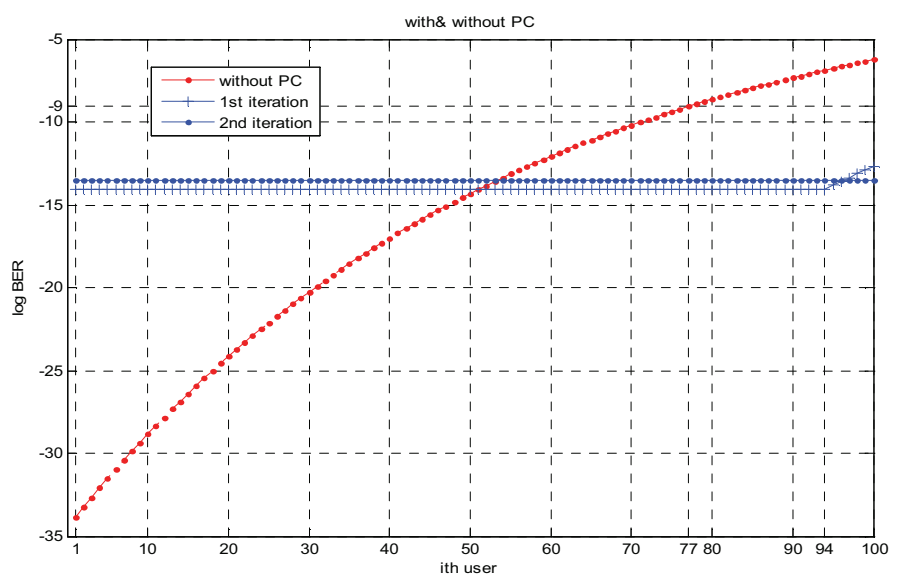

Fig. 3: Supported 100\% Users in the System with Power Control
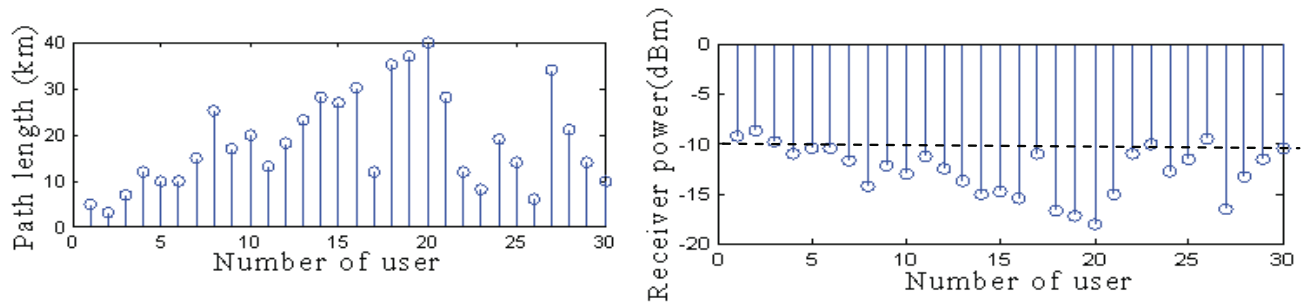

(b)

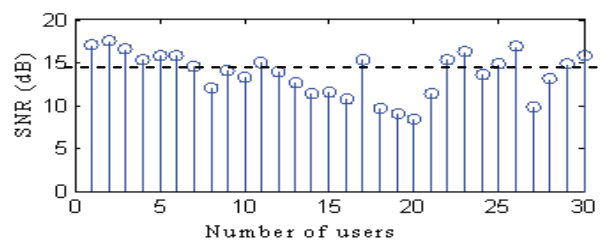

(c) 


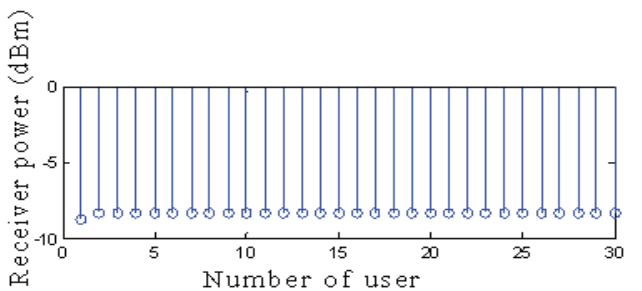

(d)

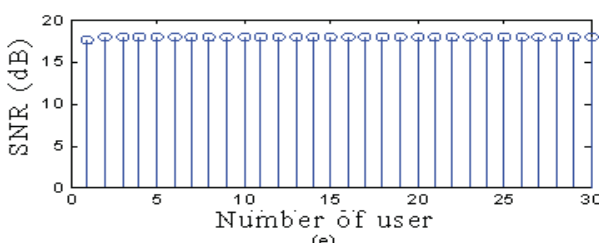

Fig. 4: (a) Random path length for 30 users (b) Received power without Power Control (c) Received power with Power Control (d) SNR with Power Control (e) SNR without Power Control

Meanwhile the distribution of random path length for 30 users is shown in Fig. 4(a), resulting to unequal received power, shown in Fig. 4(b). Note that, some users have Pr less than $-10 \mathrm{dBm}$ and therefore affected these users to have SNR lower than $16 \mathrm{~dB}$ (shown in Fig. 4(c)). Then it is depicted in Fig. 4(d-e) that, all users satisfy the target received power, resulting to SNR that less than $16 \mathrm{~dB}$ (where the BERs are at acceptable value of $10^{-9}$ ). Taking into account the noise effects of Multiple Access Interference (MAI), phase induced intensity noise (PIIN), and shot noise, the system could support 100 percent of users with power control compared to 33percent without power control when the initial transmitted power was $-1 \mathrm{dBm}$ among 30 simultaneous users.

\section{CONCLUSION}

The DFH-OCDMA is a new technique which increases the capacity of the network, but it has severe near-far problem. To control solve this problem, the power control method has been proposed to achieve optimum value for signal to noise ratio for all user. Using power control, the unwanted noises from other users could be reduced significantly. From the results, it has been shown that the system could maintain on supporting high number of users although despite of the near-far problem.

\section{REFERENCES}

[1] Salehi, J.A., Code division multiple-access techniques in optical fiber networks-Part I: Fundamental principles. IEEE Trans. Commun., 37: 824-833. 1989

[2] Salehi, J.A. and C.A. Brackett,. Code division multiple-access techniques in optical fiber networks-Part II: Systems performance analysis. IEEE Trans. Commun., 37: 834-842. 1989

[3] Dale, M.R. and R.M. Gagliardi, Channel coding for asynchronous fiber optic CDMA communications. IEEE Trans. Commun., 2485-2492. 1995.

[4] Stok, A. and E.H. Sargent, Lightning the local area: Optical code-division multiple-access and quality of service provisioning. IEEE Network Mag., 42-46. 2000.

[5] Naser G. Tarhuni, Mohamed S. Elmusrati, and Timo O. Korhonen, Nonlinear Power Control for asynchronous fiber- optic CDMA Networks. IEEE Trans. Commun., $6: 2782$ $2786,2006$.

[6] Mohammad M.N. Hamarsheh, M.H. Hossam Shalaby and Mohamad Khazani Abdullah, Design and Analysis of a Dynamic Code Division Multiple Access Communication System Based on Tunable Optical Filter. J. Light wave Technol., 23: 3959- 3965. 2005.

[7] Yashima, H. and T. Kobayashi, Optical CDMA with time hopping and power control for multimedia network. J. Lightwave Technol., 21: 695-702. 2003.

[8] Zander, J., Distributed cochannel interference control in cellular radio systems. IEEE Trans. Veh. Technol., 41: 305-311. 1992.

[9] E. Inaty,H.M.H. Shalaby, P. Fortier, and L. A. Rusch, Multirate optical fast frequency hopping CDMA system using power control. J. Lightwave Technol., 20: 166-177. 2002.

[10] Wei, Z. and Ghafouri-shiraz, Unipolar Codes with ideal inphase Cross-correlation for spectral Amplitude-Coding Optical CDMA System. IEEE Transact. Commun., 50: 1209-1212. 2002.

[11] Alexander, S.B., Optical Communication Receiver Design. 1st Edn., Spiel Optical Engineering press USA., pp: 328. 1997.

[12] Aylin Yener, Roy D. Yates and Sennur Ulukus, Interference Management for CDMA Systems through Power Control, Multi-user Detection and Beamforming. IEEE Transact. Commun., 49: 1227- 1239. 2001.

[13] Ohtsuki, T. and K. Sato, Direct-detection optical asynchronous Selected Areas Commun. IEEE J., 14: 1879-1887. 1996.

[14] E. Inaty, H. M. H. Shalaby, and P. Fortier, A new transmitterreceiver architecture for noncoherent multirate OFFH-CDMA system with fixed optimal detection threshold. IEEE J.commun.., 3: 1487-1493. 2001.

[15] E. Inaty, L. A. Rusch, and P. Fortier, Multirate optical fast frequency hopping CDMA system using power control. IEEE J.commun., 3: 1221-1227. 2000. 